\title{
Odontometric Patterns in Human Mandibular Molars for Sex Estimation in a Forensic Context
}

\author{
Sofia F. Franco ${ }^{1 *}$, Álvaro Azevedo ${ }^{2,3}$, Vítor M. J. Matos ${ }^{4}$, Daniel Mongiovi ${ }^{1}$, Alexandra \\ Teixeira $^{1}$ \\ 1 IINFACTS - Institute of Research and Advanced Training in Health Sciences and \\ Technologies, Department of Sciences, University Institute of Health Sciences \\ (IUCS), CESPU, CRL, Gandra, Portugal \\ 2 Faculty of Dental Medicine, University of Porto, Portugal \\ 3 EPIUnit - Institute of Public Health, University of Porto, Portugal \\ ${ }^{4}$ Research Centre for Anthropology and Health, Department of Life Sciences, \\ University of Coimbra, Coimbra, Portugal
}

Keywords: Sexual Dimorphism; Odontometry; Mandibular First Molar; Mandibular Canine; Forensic Odontology

\begin{abstract}
Sex estimation is an important step in the identification of human skeletal remains, since it reduces the number of potential matches to approximately one-half. The os coxae and skull are considered the most dimorphic skeletal elements; however, when unavailable, teeth may be used alternatively. This study aims to evaluate the usefulness of specific odontometric parameters from the mandibular first molar - mesiolingual-distobuccal distance (MLDB), mesiobuccal-distolingual distance (MBDL) and mesiodistal distance (MD) - on sex estimation in a Portuguese sample composed of 135 mandibles (78 males; 57 females; ages atdeath ranging from 18 to 59 years old) selected amongst the 505 individuals of the Coimbra Identified Skeletal Collection (University of Coimbra). Since canines are considered the most accurate teeth for sexual assessment, comparison between first molar parameters and canines mesiodistal dimensions (MD) was performed. Statistical analysis showed sexual dimorphism in human first molars and cut-off points between male or female groups were determined. Using the first molar MLDB and MBDL, $60.7 \%$ and $65.2 \%$ of individuals were correctly classified, respectively. Highest sex estimation accuracy was achieved with canine MD, reaching $74.6 \%$. Our results indicate that although mandibular molar dimensions are useful for sex estimation, the canine should be prioritized when available to perform this task.
\end{abstract}

In a forensic context, successful positive identification of human skeletal remains starts by determining the biological profile of the individual: sex, age at death, ancestry, and stature. Sex is usually one of the first parameters to be estimated, not only because other parameters and methods are sex dependent, but also because, when a reliable sex estimation can be obtained from the skeletal remains, only two possible outcomes are provided, male or female, thus lowering the number of possible individuals to whom the remains belong by approximately one-half (Scheuer, 2002; Scheuer and Black, 2007). In skeletal elements, sex estimation is usually obtained by studying the pelvis followed by the skull, as it is considered to yield the second highest percentage of sexual dimorphism; nonetheless, it was found, that postcranial metric measurements can perform better than those from the skull, so these should be applied when the pelvis is unavailable (Spradley \& Jantz, 2011). However, when both cranial and postcranial bones are absent or fragmented, other elements need to be considered (Bruzek \& Murail, 2006; Ubelaker, 2008; İşcan \&

*Correspondence to:

Sofia F. Franco

Institute of Research and Advanced Train-

ing in Health Sciences and Technologies,

Department of Sciences

University Institute of Health Sciences

Gandra, Portugal

E-mail: sofiafranco16@gmail.com 
Steyn, 2013). In these circumstances, dentition can be a good alternative due to its hard, stable, and durable composition. Teeth are often well preserved and can endure fire, decomposition, and severe trauma; furthermore, their analysis may contribute to the biological profile and its sexual dimorphism is represented by the differing teeth dimensions between males and females (Tabor \& Schrader, 2010; Cardoza, 2011; Vishwakarma \& Guha, 2011).

The most common tooth dimensions for sexual estimation are the mesiodistal (MD) and buccolingual (BL) crown diameters, which are defined as the distance between the most mesial and most distal points of the crown, and the distance between the most buccal and most lingual points of the tooth, respectively (White \& Folkens, 2005; İşcan \& Steyn, 2013; Kondo \& Manabe, 2016). Although most studies apply these linear dimensions, other measures, such as the Mandibular Canine Index (MCI), have also been evaluated (Scott \& Turner, 1988; Rao et al., 1989).

Of the many studies performed worldwide, most identify the canine as the most sexually dimorphic tooth of the human dentition (Acharya \& Mainali, 2007; Acharya \& Mainali, 2008; Cardoso, 2008; Zorba et al., 2011; Angadi et al., 2013; Viciano et al., 2013; Khamis et al., 2014). This is one of the reasons why the $\mathrm{MCI}$ is so often applied. For example, Rao and collaborators (1989) obtained an overall accuracy of $85.9 \%$ in sex estimation when using MCI. Nevertheless, since the degree of sexual dimorphism is population specific, some studies presented low success when employing this technique, which led to the focus being shifted to different teeth and dental dimensions (White \& Folkens, 2005; Zorba et al. 2012; İşcan \& Steyn, 2013; Narang et al., 2015).

The posterior teeth, normally having more than one root, are considered by some researchers a better choice over anterior teeth since the former are more strongly attached to the dental arch and less prone to suffer postmortem loss, thus being more frequently found in fragmentary remains (İşcan \& Steyn, 2013; Zorba et al., 2013; Narang et al., 2015). On the other hand, the linear diameters are not as easily measured in molars as they are in anterior teeth. This fact, combined with the compromise of wear and attrition on the linear diameters, guided Hillson and colleagues (2005) to come up with alternative dimensions for the posterior dentition. These included the crown diagonals: the distance between the most mesiolingual and most distobuccal points of the crown (MLDB) and distance between the most mesiobuccal and most distolingual points (MBDL).

The disarticulation of the mandible from the skull is a consequence of the decomposition of the human body and since the temporomandibular joint is one of the first to disarticulate during decay, it is not uncommon to find an isolated mandible in forensic contexts (Pinheiro, 2006; Cardoza, 2011; Cunha, 2014). In Portugal, there are very few studies on sexual dimorphism using teeth and none of them focus on the mesiodistal nor diagonal dimensions of the molars (Cardoso, 2008; Pereira et al., 2010; Gonçalves et al., 2014; Silva et al., 2016; Gouveia et al. 2017; Azevedo et al., 2019). Therefore, the aim of this study was to evaluate the sexual dimorphism in mandibular first molars using the mesiodistal and alternative diagonal dimensions and to compare it to the mandibular canine with the traditional mesiodistal distance, in a sample from the Portuguese population.

\section{Materials and Methods}

The sample was selected amongst the 505 individuals which compose the Coimbra Identified Skeletal Collection (20th century), housed at the Department of Life Sciences, University of Coimbra, according to the following selection criteria: Portuguese nationality; age at death ranging between 18 and 59 years old; mandibles presenting at least one well preserved first molar showing minimal signs of attrition (occlusal wear). The resulting study base comprised 135 human mandibles, 78 (57.8\%) belonging to males and $57(42.2 \%)$ to females.

The dimensions were taken in millimetres with a Mitutoyo Digimatic caliper, to the closest $0.01 \mathrm{~mm}$. The following dimensions were measured on the left mandibular first molars: mesiodistal distance (MD), mesiolingual to distobuccal distance (MLDB), and mesiobuccal to distolingual distance (MBDL); when left teeth were absent, the antimere was used. Additionally, MD of right canines was taken for analysis and comparison with previous measures. All dimensions were measured according to the recommendations of Hillson and collaborators (2005).

The first molar was measured in 78 male and 57 female mandibles. Only 59 out of the 135 mandibles had preserved canines ( 33 from males and 26 from females). All measurements were collected by an experienced observer, each taken in triplicate in non-consecutive order and separated in time, to avoid bias. The first value was eliminated, and the definitive value was obtained from the arithmetic average of the two last measurements. The intraobserver error was calculated with Bland-Altman analyses (Myles \& Cui, 2007). The comparison be- 
tween sexes by the selected tooth sizes were performed by the independent sample t-test, after checking the Normality assumptions by Kolmogorov-Smirnov or Shapiro-Wilk tests. Receiver Operating Characteristics (ROC) curves were applied to estimate the more accurate cut-off value for each dimension, which discriminate males from females. Tooth measurements with a value under the cut-off point were considered to belong to female individuals, and values equal to or higher than the cut-off point to belong to male individuals. Each dimension has an associated correct sexual classification accuracy, given by the sensitivity value for correctly classifying males and specificity for females.

Since MLDB and MBDL were too correlated (multicollinearity), the joint performance of sex classification was assessed using a multivariate binary logistic regression with first molar MD and canine $\mathrm{MD}$, by estimating the probability of an individual being a female. The regression model was constructed by enter-method approach. The Omnibus Test was used to check that the model holds an improvement with respect to the constant model. Additionally, goodness of fit was assessed by Hosmer-Lemeshow statistical test, and the percentage of data variance explained by the model was quantified by R-Square based statistics (Cox \& Snell and Nagelkerk). The predicted probabilities of being a female were then used as the classification variable.

For the statistical analysis, version 25.0 of SPSS software (Statistical Package for Social Sciences) was used. The level of significance was established at $5 \%$. A $p$-value with Bonferroni correction for multiple comparisons (four dimensions) of $<0.05$ was considered to indicate statistical significance.

\section{Results}

By the Bland-Altman analysis there were no relevant differences for the four dimensions (MLDB, MBDL, Molar MD and Canine MD) between the second and the third measurements, which were not statistically significant $(p>0.05)$. The maximum absolute mean difference between the second and the third measurements was $0.013 \mathrm{~mm}$ registered in the Molar MD dimension. With $95 \%$ of confidence, the mean differences for all dimensions are lower than $0.03 \mathrm{~mm}$. Moreover, the Bland-Altman analysis did not reveal a relationship between the differences obtained with the second and third measurements, and the magnitude of the measurements. It also revealed a non-dependence between the variation of those differences and the magnitude of the measurements.

Table 1 depicts the MLDB, MBDL, first Molar MD and Canine MD dimensions. All measurements were higher for males, and the mean differences were statistically significant $(p<0.05)$. In absolute terms, the larger differences $(>0.41 \mathrm{~mm})$ were found in MD dimensions.

Figure 1 shows the ROC curves obtained for MLDB and MBDL dimensions. The MBDL dimension exhibits a larger area under the curve (AUC) than that of MLDB, regardless of the cut-off considered. Figure 2 shows the ROC curves obtained for first molar MD and canine MD dimensions. This figure clearly illustrates that the AUC were similar for almost cut-offs.

Table 2 presents the results of the AUC analysis obtained for each of the four dimensions. Interestingly, the AUC values are well above 0.5 for all variables, showing statistical significance in any case $(p<0.05)$. Accordingly, as shown in Figures 1 and 2, Table 2 points out that the most discriminative variables are first molar MD and canine MD with an AUC of 0.735 and 0.801 , respectively. On the other hand, both MLDB and MBDL dimensions have much lower performance in sex discrimination (AUC values below 0.71).

The accuracy assessment in sex prediction was based on the optimal cut-offs (Table 3). Using the MBDL dimension which presented higher AUC than MLDB for all cut-offs, with the optimal cut-off of $11.44 \mathrm{~mm}, 67.9 \%$ of the males were correctly classified, whereas females were accurately identified

Table 1. Descriptive statistics for $M L D B, M B D L$, first Molar MD, and Canine MD dimensions by sex.

\begin{tabular}{ccccc}
\hline Dimensions $(\mathrm{mm})$ & $\begin{array}{c}\text { Female } \\
(\mathrm{n}=57)\end{array}$ & $\begin{array}{c}\text { Male } \\
(\mathrm{n}=78)\end{array}$ & $p$ & $\begin{array}{c}\text { 95\% CI (Difference) } \\
\text { Lower-Upper bond }\end{array}$ \\
\hline MLDB & $11.18 \pm 0.55$ & $11.51 \pm 0.56$ & 0.004 & $0.14-0.52$ \\
MBDL & $11.29 \pm 0.52$ & $11.70 \pm 0.52$ & $<0.002$ & $0.23-0.59$ \\
First Molar MD & $10.77 \pm 0.57089$ & $11.19 \pm 0.56530$ & 0.04 & $0.10-0.69$ \\
Canine MD & $6.39 \pm 0.35408$ & $6.83 \pm 0.47072$ & $<0.002$ & $0.23-0.66$ \\
\hline
\end{tabular}

Mean \pm standard deviation; $p$ represents the $p$-value with Bonferroni correction for four dimensions. 


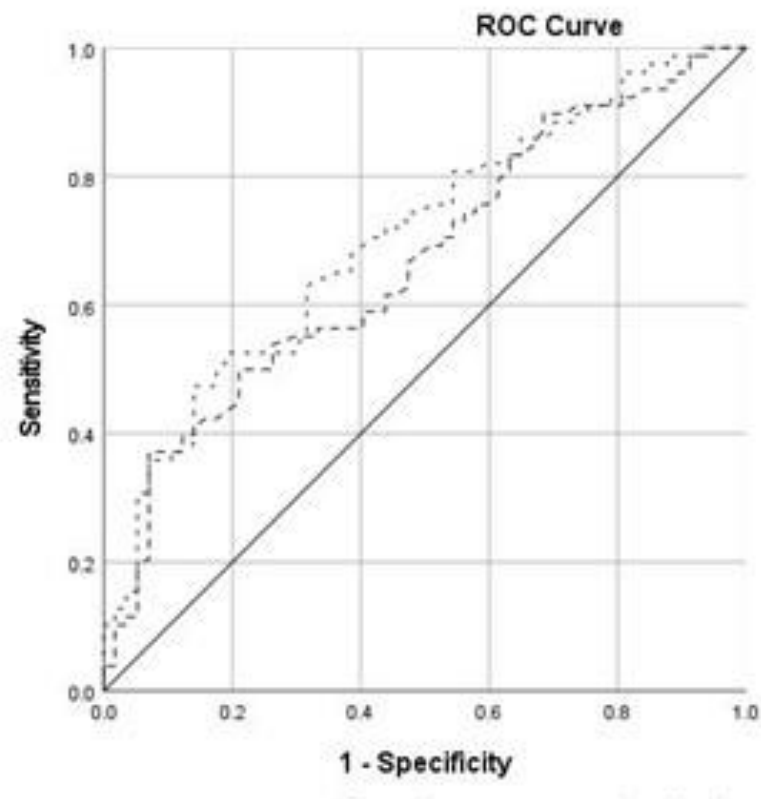

Source of the Curve

- - MLDB (mm)

$\cdots$ MBOL (mm)

- Reference Line

Diagonal segments are produced by ties.

Figure 1. ROC curve analyses for the MLDB and MBDL dimensions. The identity line represents the curve with AUC equal to 0.5 .

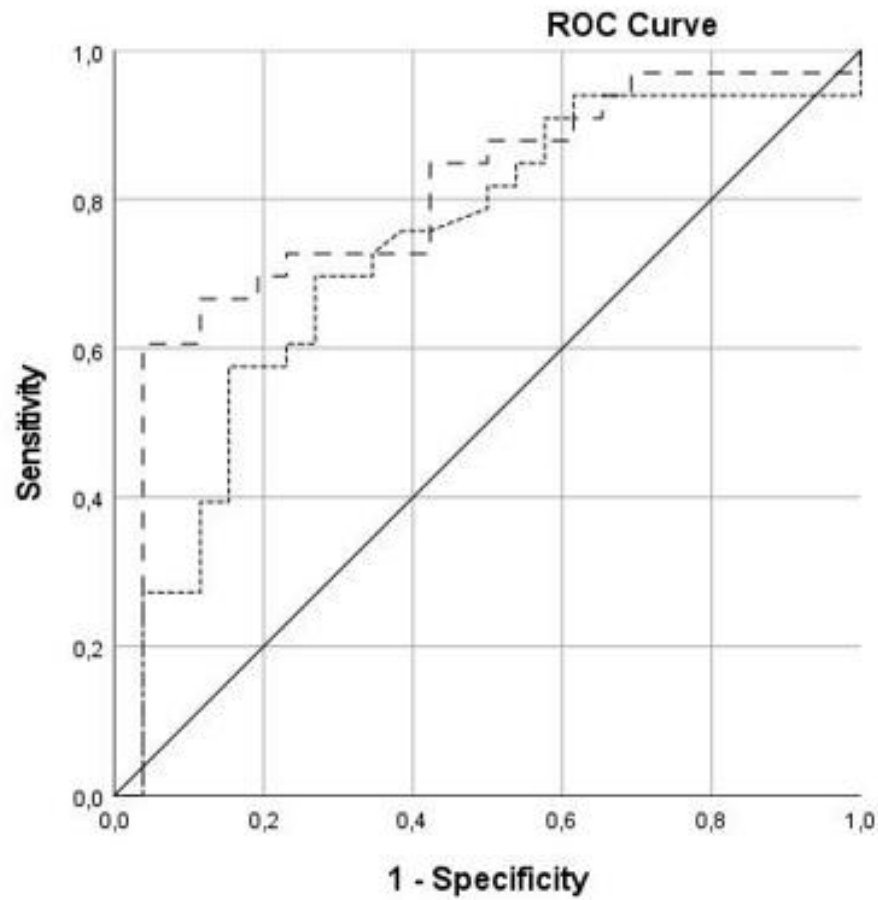

\section{Source of the Curve}

...-MD of $1^{\text {st }}$ molar (mm)

- -MD of canine (mm)

- Reference Line

Diagonal segments are produced by ties.

Figure 2. ROC curve analyses for Molar MD and Canine MD dimensions. The identity line represents the curve with AUC equal to 0.5 . 
Table 2. Area under the curve (AUC) and corresponding 95\% confidence intervals (CI) for the MLDB, MBDL, first Molar MD and Canine MD dimensions.

\begin{tabular}{cccc}
\hline Dimensions & AUC & CI $_{95 \%}$ AUC & $p$ \\
\hline MLDB (mm) & 0.668 & $0.577-0.759$ & 0.004 \\
MBDL (mm) & 0.705 & $0.617-0.792$ & $<0.002$ \\
First Molar MD (mm) & 0.735 & $0.603-0.866$ & 0.008 \\
Canine MD (mm) & 0.801 & $0.684-0.918$ & $<0.002$ \\
\hline
\end{tabular}

$p$ represents the p-value for the null hypothesis that the true AUC is 0.5 with Bonferroni correction for four dimensions.

Table 3. Overall accuracy in sex classification for the MLDB, MBDL, first Molar MD and Canine MD dimensions.

\begin{tabular}{cccc}
\hline Dimensions & & Accuracy & \\
\cline { 3 - 4 } (Optimal cut-off) & Female & Male & Total \\
\hline MLDB & $30 / 57$ & $52 / 78$ & $(62 / 135$ \\
$(11.27 m m)$ & $(52.6 \%)$ & $(66.7 \%)$ & $88 / 135$ \\
MBDL & $35 / 57$ & $53 / 78$ & $(65.2 \%)$ \\
$(11.44 m m)$ & $(61.4 \%)$ & $(67.9 \%)$ & $41 / 59$ \\
First Molar MD & $17 / 26$ & $24 / 33$ & $(69.5 \%)$ \\
$(10.89 m m)$ & $(65.4 \%)$ & $(72.7 \%)$ & $44 / 59$ \\
Canine MD (6.54mm) & $20 / 26$ & $24 / 33$ & $(74.6 \%)$ \\
First Molar & $(76.9 \%)$ & $(72.7 \%)$ & $41 / 59$ \\
MD+Canine MD* & $18 / 26$ & $23 / 33$ & $(69.5 \%)$ \\
\hline
\end{tabular}

*Multivariate binary logistic regression; the Omnibus Test of model coefficient $\left(\chi^{2}=15.502 ; \mathrm{df}=2\right.$; $p<0.001)$

in $61.4 \%$ of the cases. The corresponding overall accuracy was around $65 \%$. As expected, a greater accuracy in sex estimation was obtained with the optimal cut-offs for the first molar MD and canine MD dimensions, respectively, $10.89 \mathrm{~mm}$ and $6.54 \mathrm{~mm}$. For both dimensions, $72.7 \%$ of the males were correctly classified. Yet, the Canine MD presented better accuracy to identify the females with $76.9 \%$ accuracy, as opposed to $65.4 \%$ with the first molar MD. The Canine MD showed an overall performance of approximately $75 \%$, followed by the first Molar MD (overall accuracy around 70\%).

Multivariate binary logistic regression was then performed to assess the joint impact of the two predictors (first molar MD and canine MD) in sex classification. The Omnibus Test of model coefficient $(\chi 2=15.502 ; \mathrm{df}=2)$ was statistically significant, with $p<0.001$. Regarding the model goodness of fit, the result of the Hosmer-Lemeshow test indicates that the null hypothesis could not be rejected $(\chi 2=11.812 ; \mathrm{df}=8 ; p<0.16)$. The $\mathrm{R}$ Square based statistics was found to be $31 \%$ (Cox \& Snell statistics).
Finally, the first molar coefficient was not shown to be statistically significant $(p=0.644)$, and the coefficient estimates for canine MD $(B=-2.606$; Standard Error $=0.988$; Wald $=6.957 ; \mathrm{df}=1 ; p=0.008$ ) was found to be negative and statistically significant. So, when the first molar MD and canine MD were considered together, $69.2 \%$ of females and $69.7 \%$ of males were correctly classified, with the overall accuracy being $69.5 \%$.

\section{Discussion}

Sexual assessment is one of the first steps in the process of the identity reconstruction. When skeletal preservation is seriously compromised by taphonomic and/or anthropic reasons, teeth are of great value for sex estimation due to their strength and durability (Scheuer, 2002; Bruzek \& Murail, 2006; Scheuer and Black, 2007; Ubelaker, 2008; İscan \& Steyn, 2013; Scott et al., 2018).

Most studies estimating the sex from teeth size focus on the canines as these are known as the most sexually dimorphic teeth. In addition, it is 
considered the sturdiest and most durable tooth when faced with disease and trauma (Kaushal et al., 2003; White \& Folkens, 2005), which explains why linear dimensions and other measures like crown indexes - such as the MCI - are so commonly applied (Rao et al., 1989; Kaushal et al., 2004; Zorba et al., 2011; Khamis et al., 2014; Azevedo et al., 2019). On the other hand, its increased probability of postmortem loss, compared to posterior teeth, due to its single root led researchers to start evaluating the sexual estimation accuracy in molars (İşcan \& Steyn, 2013; Narang et al., 2015).

This study corroborates the results of previous research showing that the dentition exhibits sexual dimorphism. Statistically significant differences between males and females were found in mandibular first molars size. Furthermore, the use of molar alternative dimensions to the mesiodistal and buccolingual parameters, such as the diagonal dimensions, can be particularly useful when the teeth crowns are partially absent. In this regard, the MBDL dimension provided the highest overall estimation accuracy: $65.2 \%$ of correct classifications and an AUC of 70.5\%. However, when considering all dimensions, Canine MD was the variable with the highest correct classification accuracy, namely $74.6 \%$ and an AUC of $80.1 \%$. Both results are in accordance with the conclusions of studies from other populations, with diagonal dimensions having an accuracy range between $58.3 \%$ and $76.6 \%$ and mesiodistal dimensions a range between $63.9 \%$ and $85.8 \%$ (Karaman, 2006; Acharya \& Mainali, 2009; Acharya et al., 2011; Zorba et al., 2013; Manchanda et al., 2015; Narang et al., 2015; Tabasum et al., 2017; Azevedo et al., 2019).

When both MD variables were considered in a multivariate binary logistic regression, the accuracy of sexual estimation was $69.5 \%$, slightly lower than the value achieved with canine MD alone, and the first molar MD variable ceased to be statistically significant in this situation, which reinforces the significance of the canine. Nonetheless, these two dimensions were found to explain $31 \%$ of the existing variability between male and female individuals, which further corroborates that sexual dimorphism is present in the dentition.

Results from the first mandibular molar diagonal dimensions did not achieve a higher accuracy compared to the mesiodistal dimensions, leading to the conclusion that the canine should always be analysed when present. However, it is important to bear in mind that sample size was smaller when investigating the MD dimensions, which could have led to a slight bias of results. In this sense, the molars prove to be a good option when combined with other elements and as a corroborating method. It is also important to mention that these results only apply to a Portuguese population of the early $20^{\text {th }}$ Century since sexual dimorphism is population specific, as previously mentioned. Because sexual dimorphism varies across space and time, further studies should test this method in a contemporaneous Portuguese population (21 ${ }^{\text {st }}$ Century) to enable the investigation of secular trends in these odontometric parameters in this specific population.

\section{Conclusions}

The present results corroborate that the posterior dentition, namely odontometric parameters of the first mandibular molars, demonstrates sexual dimorphism in humans. The mesiodistal dimension of the canine was the variable that showed the highest sexual estimation accuracy, reaching levels of $74.6 \%$. The diagonal dimensions of the molar, although less accurate, proved to be acceptable variables to be used in conjunction with other dimensions when the canine is unavailable, or when partial destruction of the molar crowns makes it impossible to use other parameters. Additional research of these variables should be carried out in other populations, both to further validate the usefulness of posterior teeth in forensic scenarios and to contribute to secular trend investigations in the dentition's sexual dimorphism.

\section{Acknowledgments}

The authors would like to thank access to the Coimbra Identified Skeletal Collection provided by its curator (Prof. Sofia Wasterlain) and the Department of Life Sciences of the University of Coimbra. Thanks are also due to the Research Centre for Anthropology and Health, University of Coimbra (financed by Fundação para a Ciência e Tecnologia - FCT/MCTES, project reference UIDB/00283/2020). V.M.J.M was supported by the FCT/MCTES research project IF001862014.

\section{REFERENCES}

Acharya, A.B., Angadi, P.V., Prabhu, A., \& Nagnur, S. (2011). Validity of the mandibular canine index (MCI) in sex prediction: Reassessment in an Indian sample. Forensic Science International, 204, 207.e1-207.e4.

Acharya, A.B., \& Mainali, S. (2007). Univariate sex dimorphism in the Nepalese dentition and the 
use of discriminant functions in gender assessment. Forensic Science International, 173(1), 47-56.

Acharya, A.B., \& Mainali, S. (2008). Sex discrimination potential of buccolingual and mesiodistal tooth dimensions. Journal of Forensic Sciences, 53 (4), 790-792.

Acharya, A.B., \& Mainali, S. (2009). Limitations of the mandibular canine index in sex assessment. Journal of Forensic and Legal Medicine, 16(2), 6769.

Angadi, P.V., Hemani, S., Prabhu, S., \& Acharya, A.B. (2013). Analyses of odontometric sexual dimorphism and sex assessment accuracy on a large sample. Journal of Forensic and Legal Medicine, 20(6), 673-677.

Azevedo, A., Pereira, M.L., Gouveia, S., Tavares, J.N., \& Caldas, I.M. (2019). Sex estimation using the mandibular canine index components. Forensic Science, Medicine and Pathology, 15(2), 191197.

Bruzek, J., \& Murail, P. (2006). Methodology and Reliability of sex determination from the Sskeleton. In A. Schmitt, E. Cunha, \& J. Pinheiro (Eds.), Forensic anthropology and medicine - Complementary sciences from recovery to cause of death (pp. 225-242). Humana Press, Totowa, New Jersey.

Cardoso, H.F.V. (2008). Sample-specific (universal) metric approaches for determining the sex of immature skeletal remains using permanent tooth dimensions. Journal of Archaeological Science, 35(1), 158-168.

Cardoza, A.R. (2001). Forensic Dentistry Investigation Protocols. In C. M. Bowers (Ed.), Forensic dental evidence: An investigator's handbook (2nd ed., pp. 73-92). Elsevier Academic Press.

Cunha, E. (2014). A antropologia forense passo a passo. In A. Gomes, Enfermagem forense (Volume 1, pp. 280-288). Lidel, Edições Técnicas LDA, Lisboa.

Gonçalves, D., Granja, R., Cardoso, F.A., \& Carvalho, A.F. (2014). Sample-specific sex estimation in archaeological contexts with commingled human remains: A case study from the middle Neolithic cave of Bom Santo in Portugal. Journal of Archaeological Science, 49, 185 $-191$.

Gouveia, M.F., Santos, I.O., Santos, A.L., \& Gonçalves, D. (2017). Sample-specific odontometric sex estimation: A method with potential application to burned remains. Science and Justice, 57 (4), 262-269.

Hillson, S., FitzGerald, C., \& Flinn, H. (2005). Alternative dental measurements: Proposals and relationships with other measurements. American
Journal of Physical Anthropology, 126(4), 413-426. Işcan, M.Y., \& Steyn, M. (2013). The human skeleton in forensic medicine ( $3^{\text {rd }}$ ed). Charles C Thomas, Publisher LTD, Springfield.

Karaman, F. (2006) Use of diagonal teeth measurements in predicting gender in a Turkish population. Journal of Forensic Sciences, 51(3), 630-635.

Kaushal, S., Patnaik, V.V.G., \& Agnihotri, G. (2003). Mandibular canines in sex determination. Journal of the Anatomical Society of India, 52 (2), 119-124.

Kaushal, S., Patnaik, V.V.G., Sood, V., \& Agnihotri, G. (2004). Sex determination in North Indians using Mandibular Canine Index. Journal of Indian Academy of Forensic Medicine, 26(2), 45-49.

Khamis, M.F., Taylor, J.A., Malik, S.N., \& Townsend, G.C. (2014). Odontometric sex variation in Malaysians with application to sex prediction. Forensic Science International, 234, 183e1-183e7.

Kondo, S., \& Manabe, Y. (2016). Analytical methods and interpretation of variation in tooth morphology. Journal of Oral Biosciences, 58(3), 85-94.

Manchanda, A.S., Narang, R.S., Kahlon, S.S., \& Singh, B. (2015). Diagonal tooth measurements in sex assessment: A study on North Indian population. Journal of Forensic Dental Sciences, 7 (2), 126.

Myles PS, Cui J. Using the Bland-Altman method to measure agreement with repeated measures. British Journal of Anaesthesia, 99(3), 309-311, 2007.

Narang, R.S., Manchanda, A.S., \& Singh, B. (2015). Sex assessment by molar odontometrics in North Indian population. Journal of Forensic Dental Sciences, 7(1), 54-58.

Pereira, C., Bernardo, M., Pestana, D., Santos, J.C., \& Mendonça, M.C. (2010). Contribution of teeth in human forensic identification - Discriminant function sexing odontometrical techniques in Portuguese population. Journal of Forensic and Legal Medicine, 17(2), 105-110.

Pinheiro, J. (2006) Decay process of a cadaver. In A. Schmitt, E. Cunha, J. Pinheiro (Eds.), Forensic anthropology and medicine - Complementary sciences from recovery to cause of death (pp. 85-116). Humana Press, Totowa, New Jersey.

Rao, N.G., Rao, N.N., Pai, M.L., \& Kotian, M.S. (1989). Mandibular canine index - A clue for establishing sex identity. Forensic Science International, 42(3), 249-254.

Scheuer, L. (2002). Application of osteology to forensic medicine. Clinical anatomy: The Official Journal of the American Association of Clinical Anatomists and the British Association of Clinical Anatomists, 15(4), 297-312.

Scheuer, L., \& Black, S. (2007). Osteology. In T. 
Thompson, S. Black (Eds.), Forensic human identification: An introduction (pp. 199-219). CRC Press, Boca Raton, Florida.

Scott, G.R., \& Turner, C.G. (1988). Dental anthropology. Annual Review of Anthropology, 17(1), 99126.

Scott, G.R., Turner II, C.G., Townsend, G.C., \& Martinón-Torres, M. (2018). The anthropology of modern human teeth: dental morphology and its variation in recent and fossil Homo sapiens ( $2^{\text {nd }}$ ed). Cambridge: Cambridge University Press.

Silva, A.M., Pereira, M.L., Gouveia, S., Tavares, J.N., Azevedo, Á., \& Caldas, I.M. (2016). A new approach to sex estimation using the mandibular canine index. Medicine, Science and the Law, 56(1), 7-12.

Spradley, M.K. \& Jantz, R.L. (2011). Sex estimation in forensic anthropology: Skull versus postcranial elements. Journal of Forensic Sciences, 56(2), 289-296.

Tabasum, Q., Seharawat, J.S., Talwar, M.K., \& Pathak, R.K. (2017). Odontometric sex estimation from clinically extracted molar teeth in a North Indian population sample. Journal of Forensic Dental Sciences, 9(3), 176.

Tabor, M.P., \& Schrader, B.A. (2010). Forensic dental odentification. In D.R. Senn, \& P.G. Stimson (Eds.), Forensic dentistry (2nd ed, pp. 163-185).

CRC Press, Boca Raton, Florida.

Ubelaker, D.H. (2008). Forensic anthropology: Methodology and diversity of applications. In M.A. Katzenberg, \& S.R. Saunders (Eds.), Biological anthropology of the human skeleton ( $2^{\text {nd }}$ ed, pp. 41-69). John Wiley \& Sons, Inc., Hoboken, New Jersey.

Viciano, J., López-Lázaro, S., \& Alemán, I. (2013). Sex estimation based on deciduous and permanent dentition in a contemporary Spanish population. American Journal of Physical Anthropology, 152(1), 31-43.

Vishwakarma, N., \& Guha, R. (2011). A study of sexual dimorphism in permanent mandibular canines and its implications in forensic investigations. Nepal Medical College Journal, 13(2), 9699.

White, T.D., \& Folkens, P.A. (2005). The human bone manual. San Diego, California: Elsevier Academic Press.

Zorba, E., Moraitis, K., Eliopoulos, C., \& Spiliopoulou, C. (2012). Sex determination in modern Greeks using diagonal measurements of molar teeth. Forensic Science International, 217, 19-26.

Zorba, E., Moraitis, K., \& Manolis, S.K. Sexual dimorphism in permanent teeth of modern
Greeks. Forensic Science International, 210, 74-81.

Zorba, E., Spiliopoulou, C., \& Moraitis, K. (2013). Evaluation of the accuracy of different molar teeth measurements in assessing sex. Forensic Science, Medicine and Pathology, 9(1), 13-23. 